\title{
WEIGHTED NORM INEQUALITIES FOR BOCHNER-RIESZ SPHERICAL SUMMATION MULTIPLIERS
}

\author{
KENNETH F. ANDERSEN
}

(Communicated by Richard R. Goldberg)

\begin{abstract}
Sufficient conditions to be satisfied by nonnegative weight functions $\omega(|x|)$ are given in order that the Bochner-Riesz spherical summation multiplier operators restricted to radial functions of $R^{n}$ be bounded on $L^{p}\left(R^{n} ; \omega(|x|) d x\right)$. For a certain class of weights these conditions are also necessary.
\end{abstract}

1. Introduction. Let $\hat{f}(\xi)$ denote the Fourier transform of $f$ and let $B=$ $\{\xi:|\xi| \leq 1\}$ be the unit ball in $R^{n}$. For $\lambda \geq 0$ the Bochner-Riesz spherical summation multiplier operator $T_{\lambda}=T_{n, \lambda}$ is defined by

$$
\left(\widehat{T_{\lambda} f}\right)(\xi)=\left(1-|\xi|^{2}\right)^{\lambda} \chi_{B}(\xi) \hat{f}(\xi)
$$

and then [14] for suitable $f$

$$
\left(T_{\lambda} f\right)(x)=c_{n, \lambda} \int_{R^{n}} f(y) \frac{J_{n / 2+\lambda}(|x-y|)}{|x-y|^{n / 2+\lambda}} d y
$$

where $J_{\nu}$ is the Bessel function of the first kind of order $\nu$.

$L^{p}\left(R^{n} ; \omega(x) d x\right)$, abbreviated $L^{p}\left(R^{n}\right)$ when $\omega(x)=1$, denotes the weighted Lebesgue space of measurable functions $f$ on $R^{n}$ for which $\int_{R^{n}}|f(x)|^{p} \omega(x) d x<\infty$. The boundedness of $T_{\lambda}$ on $L^{p}$ spaces has been widely studied because of its intimate connection with the inversion of the Fourier transform by Bochner-Riesz means.

If $\lambda$ exceeds the critical index $\lambda_{c}=(n-1) / 2,(1.1)$ shows that $T_{\lambda}$ is a covolution operator with kernel in $L^{1}\left(R^{n}\right)$ so $T_{\lambda}$ is bounded on $L^{p}\left(R^{n}\right)$ for all $p, 1 \leq p \leq \infty$; moreover, since in this case $T_{\lambda} f$ is dominated by a multiple of the Hardy-Littlewood maximal function of $f[\mathbf{1 5}$, Theorem 2, p. 62], a result of B. Muckenhoupt [13] shows that $T_{\lambda}$ is bounded on $L^{p}\left(R^{n} ; \omega(x) d x\right), 1<p<\infty$, if $\omega$ satisfies

$$
\left(A_{p}\left(R^{n}\right)\right) \quad\left(\int_{Q} \omega(x) d x\right)^{1 / p}\left(\int_{Q} \omega(x)^{-p^{\prime} / p} d x\right)^{1 / p^{\prime}} \leq C \int_{Q} d x
$$

for all cubes $Q \subset R^{n}$ with sides parallel to the coordinate axis.

When $n=1, T_{0}$ reduces essentially to the Hilbert transform and hence $T_{0}$ is bounded on $L^{p}\left(R^{1} ; \omega(x) d x\right)$ if (and only if) $\omega$ satisfies $A_{p}\left(R^{1}\right)$, (see [10]) while for $n>1$ C. Fefferman [6] has shown that $T_{0}$ is bounded on $L^{p}\left(R^{n}\right)$ only if $p=2$.

Received by the editors July 30,1987 . The results of this paper were presented to the Workshop on Weighted Norm Inequalities held at the Centre de Recherches Mathématiques, Université de Montréal, October 29-31, 1987.

1980 Mathematics Subject Classification (1985 Revision). Primary 42B15, 42B20, 44A15.

Research supported in part by NSERC grant \#A-8185. 
For $0<\lambda \leq \lambda_{c}$, the boundedness properties of $T_{\lambda}$ are not yet fully understood. Herz [8] has shown that $T_{\lambda}$ is unbounded if $p \leq p_{0}$ or $p \geq p_{0}^{\prime}$ where $p_{0}=p_{0}(\lambda, n)=$ $2 n /(n+1+2 \lambda)$ and it has been conjectured that $T_{\lambda}$ is bounded for $p_{0}<p<p_{0}^{\prime}$. The conjecture has been verified for $n=2$ by L. Carleson and P. Sjölin [3] but for $n \geq 3$ it has been verified only for $\lambda>(n-1) / 2(n+1)$, see $[\mathbf{5}, \mathbf{7}, \mathbf{1 7}]$.

The restriction of $T_{\lambda}$ to radial functions in $R^{n}$ is bounded on $L^{p}$ for $p_{0}<p<p_{0}^{\prime}$, $0 \leq \lambda \leq \lambda_{c}$, see $[8,19,4]$. S. Chanillo and B. Muckenhoupt [4] proved weak type $\left(p_{0}, p_{0}\right)$ estimates for the restricted operator when $0<\lambda \leq \lambda_{c}$ but as C. Kenig and P. Tomas [11] have shown, these do not extend to the case $\lambda=0$ when $n>1$.

I. Hirschman, Jr. [9] obtained the boundedness of $T_{\lambda}, 0 \leq \lambda<\lambda_{c}$, on the power weighted space $L^{2}\left(R^{n} ;|x|^{\alpha} d x\right)$ for $|\alpha|<1+2 \lambda$.

The main result of this paper gives sufficient conditions on the weight function $\omega$ which ensure that $T_{\lambda}$ restricted to radial functions is bounded on $L^{p}\left(R^{n} ; \omega(|x|) d x\right)$. For a certain class of weights of the form $\omega(r)=r^{\alpha}(1+r)^{\beta-\alpha}$ these conditions are also seen to be necessary. These results generalize those of [1] where the case $\lambda=0$ was treated.

THEOREM 1. Let $1<p<\infty, 1 / p+1 / p^{\prime}=1, \lambda>0$ and suppose $\omega(|x|)$ is nonnegative on $R^{n}$ and satisfies

$$
\left(\int_{a}^{b} \frac{\omega(r) r^{n-1}}{(1+r)^{(n-1-2 \lambda) p / 2}} d r\right)^{1 / p}\left(\int_{a}^{b} \frac{\omega(r)^{-p^{\prime} / p} r^{n-1}}{(1+r)^{(n-1-2 \lambda) p^{\prime} / 2}} d r\right)^{1 / p^{\prime}}
$$

$$
\leq K \int_{a}^{b} \frac{r^{n-1}}{(1+r)^{n-1-2 \lambda}} d r
$$

for some constant $K=K_{n, \lambda, p, \omega}$ and all $0 \leq a<b<\infty$. Then there is a constant $C=C_{n, \lambda, p, K}$ such that

$$
\int_{R^{n}}\left|\left(T_{n, \lambda} f\right)(x)\right|^{p} \omega(|x|) d x \leq C \int_{R^{n}}|f(x)|^{p} \omega(|x|) d x
$$

for all radial functions $f$ in $L^{p}\left(R^{n} ; \omega(|x|) d x\right)$.

COROLLARY. If $-n<\alpha<n(p-1)$ then (1.3) holds with $\omega(r)=r^{\alpha}(1+r)^{\beta-\alpha}$ if and only if

$$
-n+(n-1-2 \lambda) p / 2<\beta<-n+(n+1+2 \lambda) p / 2 .
$$

The corollary may be proved as follows. It is easily verified that (1.2) holds with $\omega(r)=r^{\alpha}(1+r)^{\beta-\alpha}$ if $-n<\alpha<n(p-1)$ and $\beta$ satisfies (1.4) so the sufficiency follows directly from the theorem. Since $\left|\left(T_{\lambda} \chi_{B}\right)(x)\right| \sim c_{n, \lambda}|x|^{-(n+1+2 \lambda) / 2}$ as $|x| \rightarrow$ $\infty$, and $\chi_{B} \in L^{p}\left(R^{n} ; \omega(|x|) d x\right)$ for $\alpha>-n$, (1.3) with $f=\chi_{B}$ shows the necessity of the upper bound for $\beta$ in (1.4). On the other hand, $\chi_{B} \in L^{p^{\prime}}\left(R^{n} ; \omega(|x|)^{-p^{\prime} / p} d x\right)$ for $\alpha<n(p-1)$ so a standard duality argument then shows the necessity of the lower bound for $\beta$ in (1.4).

Note that with $\alpha=\beta=0$ the corollary recovers the $L^{p}\left(R^{n}\right)$ boundedness result for $p_{0}<p<p_{0}^{\prime}$ cited above while with $p=2$ and $\alpha=\beta$ (1.4) coincides with the range obtained by Hirschman for the unrestricted operator. 
Let $\Omega(n, \lambda, p)$ denote the class of $\omega$ which satisfy (1.2). The following properties are easily verified:

$$
\begin{aligned}
& n_{1}<n_{2} \quad \Rightarrow \quad \Omega\left(n_{1}, \lambda, 2\right) \subset \Omega\left(n_{2}, \lambda, 2\right), \\
& \lambda_{1}<\lambda_{2} \quad \Rightarrow \quad \Omega\left(n, \lambda_{1}, p\right) \subset \Omega\left(n, \lambda_{2}, p\right), \\
& p_{1}<p_{2} \quad \Rightarrow \quad \Omega\left(n, \lambda_{c}, p_{1}\right) \subset \Omega\left(n, \lambda_{c}, p_{2}\right) .
\end{aligned}
$$

The examples provided by the corollary show that the analogues of (1.5) for $p \neq 2$ and of (1.7) for $\lambda \neq \lambda_{c}$ do not hold. Note also that if $\omega(r)=r^{\alpha}$ then $\omega \in \Omega\left(n, \lambda_{c}, p\right)$ if and only if $\omega(|x|)$ satisfies $A_{p}\left(R^{n}\right)$.

Theorem 1 may be generalized somewhat as follows. For radial functions $f(x)=$ $f_{0}(|x|)$ on $R^{n},\left(T_{n, \lambda} f\right)(x)=\left(R_{n, \lambda} f_{0}\right)(|x|)$ is also radial and if $P_{k}(x)$ is a harmonic homogeneous polynomial of degree $k$ in $R^{n}$, then

$$
\left(T_{n, \lambda} f P_{k}\right)(x)=(-1)^{k}\left(R_{n+2 k, \lambda} f_{0}\right)(|x|) P_{k}(x),
$$

see [16, Chapter IV, $\S \S 2,3]$. This with Theorem 1 yields Theorem 2.

THEOREM 2. Let $1<p<\infty, 1 / p+1 / p^{\prime}=1, \lambda>0, k \geq 0$ an integer and suppose $\omega(|x|)$ is nonnegative on $R^{n}$ and satisfies

$$
\begin{gathered}
\left(\int_{a}^{b} \frac{\omega(r) r^{k p+n-1}}{(1+r)^{(n+2 k-1-2 \lambda) p / 2}} d r\right)^{1 / p}\left(\int_{a}^{b} \frac{\omega(r)^{-p^{\prime} / p} r^{k p^{\prime}+n-1}}{(1+r)^{(n+2 k-1-2 \lambda) p^{\prime} / 2}} d r\right)^{1 / p^{\prime}} \\
\leq K \int_{a}^{b} \frac{r^{n+2 k-1}}{(1+r)^{n+2 k-1-2 \lambda}} d r
\end{gathered}
$$

for some constant $K=K_{n, \lambda, p, \omega}$ and all $0 \leq a<b<\infty$. Then there is a constant $C=C_{n, \lambda, p, k, K}$ such that

$$
\int_{R^{n}}\left|\left(T_{n, \lambda} f P_{k}\right)(x)\right|^{p} \omega(|x|) d x \leq C \int_{R^{n}}\left|f(x) P_{k}(x)\right|^{p} \omega(|x|) d x
$$

for all radial functions $f$ and all harmonic homogeneous polynomials $P_{k}$ of degree $k$ in $R^{n}$ with $f P_{k}$ in $L^{p}\left(R^{n} ; \omega(|x|) d x\right)$.

2. Proof of Theorem 1. We follow the usual practice that $C$ will denote an absolute constant, not necessarily the same from line to line. The proof requires the following lemmas. Define the operators $P=P_{n, \lambda}, Q=Q_{n, \lambda}$, and $M$ for $s>0$ by

$$
\begin{gathered}
(P \phi)(s)=(1+s)^{-(n+1+2 \lambda) / 2} \int_{0}^{s}(1+r)^{-(n-1) / 2} \phi(r) r^{n-1} d r \\
(Q \phi)(s)=(1+s)^{-(n-1) / 2} \int_{s}^{\infty}(1+r)^{-(n+1+2 \lambda) / 2} \phi(r) r^{n-1} d r \\
(M \phi)(s)=\sup _{0<h<s} \frac{1}{2 h} \int_{s-h}^{s+h}|\phi(r)| d r
\end{gathered}
$$

LEMMA 1. If $\lambda>0$ and $f(x)=f_{0}(|x|)$ is a simple radial function on $R^{n}$ then there is a constant $C=C_{n, \lambda}$ such that

$$
\left|\left(T_{\lambda} f\right)(x)\right| \leq C\left\{\left([P+Q]\left|f_{0}\right|\right)(|x|)+\left(M f_{0} \chi_{[|x| / 2,2|x|]}\right)(|x|)\right\} .
$$

ProOF. Set $|x|=s,|y|=r$ and consider separately the cases $s \leq 4$ and $s>4$. 
If $s \leq 4,(1.1)$ and the estimates [18, pp. 49, 199]

$$
\left|J_{\nu}(t)\right| \leq C_{\nu} t^{\nu}, \quad\left|J_{\nu}(t)\right| \leq C_{\nu}(1+t)^{-1 / 2}, \quad t>0,
$$

show that $\left|\left(T_{\lambda} f\right)(x)\right|$ does not exceed

$$
C_{n, \lambda}\left\{\int_{0}^{8}\left|f_{0}(r)\right| r^{n-1} d r+\int_{8}^{\infty}(1+r)^{-(n+1+2 \lambda) / 2}\left|f_{0}(r)\right| r^{n-1} d r\right\}
$$

since $s \leq 4$ and $r \geq 8$ implies $|x-y| \geq r-s \geq r / 2 \geq(1+r) / 4$. Thus $\left|\left(T_{\lambda} f\right)(x)\right| \leq$ $C_{n, \lambda}\left\{\left([P+Q]\left|f_{0}\right|\right)(s)\right\}$ for $s \leq 4$.

Now let $s>4$ and set $f=f_{1}+f_{2}$, where $f_{1}(y)=\left(f_{0} \chi_{(0,2)}\right)(|y|)$. Then (1.1) and (2.2) show that

$$
\left|\left(T_{\lambda} f_{1}\right)(x)\right| \leq C_{n, \lambda} \int_{0}^{2}(1+s)^{-(n+1+2 \lambda) / 2}\left|f_{0}(r)\right| r^{n-1} d r \leq C_{n, \lambda}\left(P\left|f_{0}\right|\right)(s)
$$

since in this case $|x-y| \geq s-r \geq s / 2 \geq(1+s) / 4$. The estimates (3.3), (3.4) and (3.5) of $\left[4\right.$, p. 701] show that $\left|\left(T_{\lambda} f_{2}\right)(x)\right|$ does not exceed a constant multiple of

$$
\begin{aligned}
s^{-(n+1+2 \lambda) / 2} & \int_{2}^{s / 2}(1+r)^{-(n-1) / 2}\left|f_{0}(r)\right| r^{n-1} d r \\
& +s^{-(n-1) / 2} \int_{2 s}^{\infty}(1+r)^{-(n+1+2 \lambda) / 2}\left|f_{0}(r)\right| r^{n-1} d r \\
& +\left(M f_{0} \chi_{[s / 2,2 s]}\right)(s)
\end{aligned}
$$

and this is bounded by

$$
C_{n, \lambda}\left\{\left([P+Q]\left|f_{0}\right|\right)(s)+\left(M f_{0} \chi_{[s / 2,2 s]}\right)(s)\right\} .
$$

Thus, $\left|\left(T_{\lambda} f\right)(x)\right| \leq\left|\left(T_{\lambda} f_{1}\right)(x)\right|+\left|\left(T_{\lambda} f_{2}\right)(x)\right|$ shows that $(2.1)$ holds for $s>4$ also. This proves Lemma 1 .

LEMMA 2. Let $1<p<\infty, 1 / p+1 / p^{\prime}=1$ and suppose $\omega \geq 0$ on $(0, \infty)$. The following statements are equivalent:

(a) There is a constant $C=C_{n, \lambda, p, \omega}$ such that

$$
\int_{0}^{\infty}|(P \phi)(s)|^{p} \omega(s) s^{n-1} d s \leq C \int_{0}^{\infty}|\phi(r)|^{p} \omega(r) r^{n-1} d r .
$$

(b) There is a constant $C=C_{n, \lambda, p, \omega}$ such that

$$
\int_{0}^{\infty}|(Q \psi)(s)|^{p^{\prime}} \omega(s)^{-p^{\prime} / p} s^{n-1} d s \leq C \int_{0}^{\infty}|\psi(r)|^{p^{\prime}} \omega(r)^{-p^{\prime} / p} r^{n-1} d r .
$$

(c) There is a constant $C=C_{n, \lambda, p, \omega}$ such that for all $a>0$

$$
\left(\int_{a}^{\infty} \frac{\omega(r) r^{n-1}}{(1+r)^{(n+1+2 \lambda) p / 2}} d r\right)^{1 / p}\left(\int_{0}^{a} \frac{\omega(r)^{-p^{\prime} / p} r^{n-1}}{(1+r)^{(n-1) p^{\prime} / 2}} d r\right)^{1 / p^{\prime}} \leq C .
$$

PROOF. Since $\int_{0}^{\infty} \psi(s)(P \phi)(s) s^{n-1} d s=\int_{0}^{\infty} \phi(r)(Q \psi)(r) r^{n-1} d r$ for $\phi, \psi \geq 0$, a standard duality argument shows that (a) and (b) are equivalent. Theorem 1 of [12] yields the equivalence of (a) and (c). 
LEMMA 3. Let $1<p<\infty, a_{0} \geq 0$, and $V(r) \geq 0$. If for some $\varepsilon>0$ there is a constant $C=C_{p, a_{0}, \varepsilon, V}$ such that for all $a>a_{0}$

$$
\left(\int_{a}^{\infty}\left(\frac{a}{r}\right)^{\epsilon} \frac{V(r)}{r^{p}} d r\right)^{1 / p}\left(\int_{0}^{a} V(r)^{-p^{\prime} / p} d r\right)^{1 / p^{\prime}} \leq C
$$

then there is a constant $C=C_{p, a_{0}, \varepsilon, V}$ such that for all $a>a_{0}$

$$
\left(\int_{a}^{\infty} \frac{V(r)}{r^{p}} d r\right)^{1 / p}\left(\int_{0}^{a} V(r)^{-p^{\prime} / p} d r\right)^{1 / p^{\prime}} \leq C .
$$

PROOF. This is proved in [2, Lemma 2] for the case $a_{0}=0$; the general case is entirely similar and is therefore omitted.

Returning to the proof of Theorem 1, observe first that (1.2) shows $\omega$ and $\omega^{-p^{\prime} / p}$ are locally integrable on $[0, \infty)$. Hence the set of simple functions is dense in both $L^{p}\left(R^{n} ; \omega(|x|) d x\right)$ and $L^{p^{\prime}}\left(R^{n} ; \omega(|x|)^{-p^{\prime} / p} d x\right)$ and therefore by Lemma 1 it suffices to show that

$$
\begin{aligned}
& \int_{0}^{\infty}\left|\left(P f_{0}\right)(s)\right|^{p} \omega(s) s^{n-1} d s \\
& \int_{0}^{\infty}\left|\left(Q f_{0}\right)(s)\right|^{p} \omega(s) s^{n-1} d s
\end{aligned}
$$

and

$$
\int_{0}^{\infty}\left|\left(M f_{0} \chi_{[s / 2,2 s]}\right)(s)\right|^{p} \omega(s) s^{n-1} d s
$$

are each bounded by a constant multiple of $\int_{0}^{\infty}\left|f_{0}(r)\right|^{p} \omega(r) r^{n-1} d r$.

Consider (2.3) first. For $t>a>0,(1.2)$ implies there is a constant $C=C_{n, \lambda, p, K}$ such that

$$
\begin{aligned}
& \left(\int_{a}^{t} \frac{\omega(r) r^{n-1}}{(1+r)^{(n-1-2 \lambda) p / 2}} d r\right)\left(\int_{0}^{a} \frac{\omega(r)^{-p^{\prime} / p} r^{n-1}}{(1+r)^{(n-1-2 \lambda) p^{\prime} / 2}} d r\right)^{p / p^{\prime}} \\
& \quad \leq K^{p}\left(\int_{0}^{t} \frac{r^{n-1}}{(1+r)^{n-1-2 \lambda}} d r\right)^{p} \leq C(1+t)^{(2 \lambda+1) p}
\end{aligned}
$$

Multiplying this by $(1+t)^{-(2 \lambda+1) p-2}$, integrating the result over $a<t<\infty$ and using Fubini's theorem on the left shows that

$$
\left(\int_{a}^{\infty}\left(\frac{1+a}{1+r}\right) \frac{\omega(r) r^{n-1}}{(1+r)^{(n+1+2 \lambda) p / 2}} d r\right)\left(\int_{0}^{a} \frac{\omega(r)^{-p^{\prime} / p} r^{n-1}}{(1+r)^{(n-1-2 \lambda) p^{\prime} / 2}} d r\right)^{p / p^{\prime}}
$$

is bounded by a constant $C$. Thus, Lemma 3 shows that

$$
\left(\int_{a}^{\infty} \frac{\omega(r) r^{n-1}}{(1+r)^{(n+1+2 \lambda) p / 2}} d r\right)^{1 / p}\left(\int_{0}^{a} \frac{\omega(r)^{-p^{\prime} / p} r^{n-1}}{(1+r)^{(n-1-2 \lambda) p^{\prime} / 2}} d r\right)^{1 / p^{\prime}}
$$

is bounded by $C$ for all $a \geq 1$. If $a<1$, the first factor of $(2.6)$ is bounded by a constant times the sum of

$$
\left(\frac{1}{(1+a)^{(1+2 \lambda) p}} \int_{a}^{1} \frac{\omega(r) r^{n-1}}{(1+r)^{(n-1-2 \lambda) p / 2}} d r\right)^{1 / p}
$$


and

$$
\left(\int_{1}^{\infty} \frac{\omega(r) r^{n-1}}{(1+r)^{(n+1+2 \lambda) p / 2}} d r\right)^{1 / p}
$$

Thus, for $a<1,(2.6)$ is bounded by the sum of two terms, the first of which is bounded because of (1.2) while the second is bounded because of (2.6) with $a=1$. Thus for $a>0$ we have

$$
\left(\int_{a}^{\infty} \frac{\omega(r) r^{n-1}}{(1+r)^{(n+1+2 \lambda) p / 2}} d r\right)^{1 / p}\left(\int_{0}^{a} \frac{\omega(r)^{-p^{\prime} / p} r^{n-1}}{(1+r)^{(n-1-2 \lambda) p^{\prime} / 2}} d r\right)^{1 / p^{\prime}} \leq C
$$

so parts (a) and (c) of Lemma 2 show that (2.3) has the required bound.

That (2.4) has the required bound now follows easily from the duality expressed by parts (a) and (b) of Lemma 2 since (1.2) is self-dual in the sense that $\omega$ satisfies (1.2) for a given $p$ if and only if (1.2) is satisfied with $\omega$ and $p$ replaced by $\omega^{-p^{\prime} / p}$ and $p^{\prime}$.

Finally, to show that (2.5) has the required bound, observe first that (1.2) shows there is a constant $C=C_{n, \lambda, K}$ independent of the integer $m$ such that

$$
\left(\int_{a}^{b} \omega(r) d r\right)^{1 / p}\left(\int_{a}^{b} \omega(r)^{-p^{\prime} / p} d r\right)^{1 / p^{\prime}} \leq C(b-a)
$$

whenever $2^{m-1} \leq a<b \leq 2^{m+2}$. Thus Muckenhoupt's well-known result [13] for the Hardy-Littlewood maximal function shows that

$$
\int_{2^{m-1}}^{2^{m+2}}|(M \phi)(s)|^{p} \omega(s) d s \leq C \int_{2^{m-1}}^{2^{m+2}}|\phi(r)|^{p} \omega(r) d r
$$

for a constant $C=C_{n, \lambda, K}$ independent of $m$. Hence (2.5) is bounded by

$$
\begin{aligned}
& \sum_{m=-\infty}^{\infty} \int_{2^{m}}^{2^{m+1}}\left|\left(M \phi \chi_{\left[2^{m-1}, 2^{m+2}\right]}\right)(s)\right|^{p} \omega(s) s^{n-1} d s \\
& \quad \leq C \sum_{m=-\infty}^{\infty} 2^{(m+1)(n-1)} \int_{2^{m-1}}^{2^{m+2}}|\phi(r)|^{p} \omega(r) d r \\
& \leq C \int_{0}^{\infty}|\phi(r)|^{p} \omega(r) r^{n-1} d r
\end{aligned}
$$

as required. This completes the proof of Theorem 1 .

\section{REFERENCES}

1. K. F. Andersen, Weighted inequalities for the disc multiplier, Proc. Amer. Math. Soc. 83 (1981), 269-275.

2. K. F. Andersen and B. Muckenhoupt, Weighted weak type Hardy inequalities with applications to Hilbert transforms and maximal functions, Studia Math. 72 (1982), 9-26.

3. L. Carleson and P. Sjölin, Oscillatory integrals and a multiplier problem for the disc, Studia Math. 44 (1972), 287-299.

4. S. Chanillo and B. Muckenhoupt, Weak type estimates for Bochner-Riesz spherical summation multipliers, Trans. Amer. Math. Soc. 294 (1986), 693-703.

5. C. Fefferman, Inequalities for strongly singular convolution operators, Acta Math. 124 (1970), 9-36.

6. __ The multiplier problem for the ball, Ann. of Math. 94 (1971), 330-336. 
7. __ A note on spherical summation multipliers, Israel J. Math. 15 (1973), 44-52.

8. C. Herz, On the mean inversion of Fourier and Hankel transforms, Proc. Nat. Acad. Sci. U.S.A. 40 (1954), 996-999.

9. I. I. Hirschman, Jr., Multiplier transformations II, Duke Math. J. 28 (1961), 45-56.

10. R. Hunt, B. Muckenhoupt and R. Wheeden, Weighted norm inequalities for the conjugate function and Hilbert transform, Trans. Amer. Math. Soc. 176 (1973), 227-251.

11. C. Kenig and P. Tomas, The weak behavior of spherical means, Proc. Amer. Math. Soc. 78 (1980), 48-50.

12. B. Muckenhoupt, Hardy's inequalities with weights, Studia Math. 44 (1972), 31-38.

13. __ Weighted norm inequalities for the Hardy maximal function, Trans. Amer. Math. Soc. 165 (1972), 207-226.

14. E. M. Stein, Interpolation of linear operators, Trans. Amer. Math. Soc. 83 (1956), 482-496.

15. __ Singular integrals and the differentiability properties of functions, Princeton Univ. Press, Princeton, N.J., 1970.

16. E. M. Stein and G. Weiss, Introduction to Fourier analysis on Euclidean spaces, Princeton Univ. Press, Princeton, N.J., 1971.

17. P. Tomas, Restriction theorems for the Fourier transform, Harmonic Analysis on Euclidean Space (G. Weiss and S. Wainger, Eds.), Proc. Sympos. Pure Math., vol 35, Part I, Amer. Math. Soc., Providence, R.I., 1979, pp. 111-114.

18. G. N. Watson, A treatise on the theory of Bessel functions, 2nd ed., Cambridge Univ. Press, London and New York, 1966.

19. G. V. Welland, Norm convergence of Riesz-Bochner means for radial functions, Canad. J. Math. 27 (1975), 176-185.

Department of Mathematics, University of Alberta, Edmonton, Alberta, CANADA T6G 2G1 